PROCEEDINGS OF THE

AMERICAN MATHEMATICAL SOCIETY

Volume 127, Number 7, Pages 2175-2182

S 0002-9939(99)04868-6

Article electronically published on March 1, 1999

\title{
ON EQUIVARIANT SLICE KNOTS
}

\author{
JAE CHOON CHA AND KI HYOUNG KO
}

(Communicated by Ronald A. Fintushel)

\begin{abstract}
We suggest a method to detect that two periodic knots are not equivariantly concordant, using surgery on factor links. We construct examples which satisfy all known necessary conditions for equivariant slice knotsNaik's and Choi-Ko-Song's improvements of classical results on Seifert forms and Casson-Gordon invariants of slice knots - but are not equivariantly slice.
\end{abstract}

\section{INTRODUCTION}

An oriented $\operatorname{knot} \mathcal{K}$ in $S^{3}$ is called a periodic knot of period $p$, or a $p$-periodic $k n o t$ if there is an orientation preserving action of the cyclic group $\mathbf{Z}_{p}$ on $S^{3}$ whose fixed point set is a circle (called an axis) disjoint to $\mathcal{K}$, and $\mathcal{K}$ is invariant under the action. By the confirmed Smith conjecture, we may assume the cyclic group $\mathbf{Z}_{p}$ is generated by the standard $(2 \pi / p)$-rotation around an unknotted axis. It is not known whether a knot can be invariant under more than one $\mathbf{Z}_{p}$-actions. In this paper we regard a $p$-periodic knot as a knot equipped with the $\mathbf{Z}_{p}$-action around a fixed axis, denoted by $\mathcal{A}$.

Two knots $K_{0}$ and $K_{1}$ are called concordant if there is a proper submanifold $C$ in $S^{3} \times[0,1]$ diffeomorphic to $S^{1} \times[0,1]$ such that $C \cap\left(S^{3} \times 0\right)=K_{0}, C \cap\left(S^{3} \times 1\right)=-K_{1}$. $C$ is called a concordance between $K_{0}$ and $K_{1}$. Two $p$-periodic knots $\mathcal{K}_{0}$ and $\mathcal{K}_{1}$ are equivariantly concordant if there is a concordance $\mathcal{C}$ which is invariant under an extended $\mathbf{Z}_{p}$-action on $S^{3} \times[0,1]$ that extends the standard $\mathbf{Z}_{p}$-actions on $S^{3} \times 0$ and on $S^{3} \times 1$ with the fixed axis. An extended $\mathbf{Z}_{p}$-action on $S^{3} \times[0,1]$ is sometimes required to be standard so that it fixes $\mathcal{A} \times[0,1]$ [4]. Since there is a $\mathbf{Z}_{p}$-action on $S^{4}$ that fixes a knotted $S^{2}[6]$, this restriction seemingly gives a stronger relation than the equivariant concordance defined here.

A knot is called a slice knot if it is concordant to the unknot. The unknot that forms a hopf link with the axis $\mathcal{A}$ can be considered as a p-periodic knot in an obvious way, and a periodic knot is called equivariantly slice if it is equivariantly concordant to the unknot.

In the case of knots without periodicity, many invariants under knot concordance are known. One of the most important invariants is the Seifert form. In [11], J. Levine showed that the Seifert form of a slice knot admits a metabolizer. In the case of odd higher dimensional knots, he also showed that the converse is true. However, in the case of 1-dimensional knots, a secondary obstruction is found by

Received by the editors September 21, 1997.

1991 Mathematics Subject Classification. Primary 57M25, 57M60; Secondary 57Q60.

Key words and phrases. Periodic knot, concordance.

(C)1999 American Mathematical Society 
A. Casson and C. Gordon [1, 2]. Using it, they showed that there are non-slice knots whose Seifert forms admit metabolizers.

These results for knot concordance without periodicity have been applied to equivariant knot concordance. Let $\mathcal{K}$ be a $p$-periodic knot. In [14], S. Naik showed that $\mathcal{K}$ admits an equivariant Seifert surface $F$ with respect to the $\mathbf{Z}_{p}$-action $f$, that is, $f(F)=F$. Naik also showed that if $\mathcal{K}$ is equivariantly slice, then there is a metabolizer $H \subset H_{1}(F)$ of the Seifert form $\theta: H_{1}(F) \times H_{1}(F) \rightarrow \mathbf{Z}$ which is equivariant with respect to the induced action on $H_{1}(F)$, that is, $2 \operatorname{rank} H=$ $\operatorname{rank} H_{1}(F), \theta(H \times H)=0$, and $\left(\left.f\right|_{F}\right)_{*}(H)=H$.

The Casson-Gordon invariant is also applied to periodic knots as follows. In [8], the group of characters on the first homology of the $d$-fold cyclic cover of $S^{3}$ branched along $\mathcal{K}$ is identified with a certain subgroup $A^{d}$ of $H_{1}(F) \otimes \mathbf{Q} / \mathbf{Z}$. In [14], it is proved that for an equivariant slice knot $\mathcal{K}$, there is an equivariant metabolizer $H$ in the above sense such that if $d$ is a prime power order and $\chi \in A^{d} \cap H \otimes \mathbf{Q} / \mathbf{Z}$ is a character of prime power order, then the Casson-Gordon invariant $\tau(\mathcal{K}, \chi)$ vanish. We remark that Naik also obtained necessary conditions on Casson-Gordon invariants and Alexander polynomials (see [14, Section 5]) that are weaker but do not depend on the choice of $\mathbf{Z}_{p}$ actions for $\mathcal{K}$.

On the other hand, in [4], K. Ko, D. Choi and W. Song defined an equivalence relation on Seifert matrices of periodic knots, and showed that Seifert matrices of two equivariantly concordant knots are equivalent in their sense. That equivalence relation of Seifert matrices of periodic knots is a strictly finer refinement of Levine's matrix cobordism relation, and the results of [4] make visible in a very rigid form the shape of Naik's equivariant metabolizer. Moreover, the results of [4] can be applied to detect not only that a periodic knot is not equivariantly slice, but also that two periodic knots are not equivariantly concordant.

In this manuscript, we will investigate concordance of periodic knots using factor knots, which are obtained by taking quotients of the ambient sphere modulo $\mathbf{Z}_{p^{-}}$ actions of periodic knots. Our main result is the following.

Theorem 1. There are periodic knots which admit equivariant metabolizers in the sense of $[14,4]$, and have trivial Casson-Gordon invariants for the characters corresponding to equivariant metabolizers, but are not equivariantly slice.

In other words, Naik's condition [14] and Choi-Ko-Song's condition [4] are not sufficient for a knot to be equivariantly slice or for two knots to be equivariantly concordant. We will construct examples of such periodic knots.

\section{EQUIVARIANT CONCORDANCES}

Factor knots of periodic knots. From now on, we fix a period $p$ of periodic knots. Let $\mathcal{K}$ be a periodic knot in $S^{3}$. The quotient space of $S^{3}$ modulo the $\mathbf{Z}_{p^{-}}$ action is again $S^{3}$, and the image of $\mathcal{K}$ in the quotient space is again a (knotted) circle $K$. We will call $K$ the factor knot of $\mathcal{K}$. On the other hand, the image of the axis $\mathcal{A}$ in the quotient space is again an unknotted circle, which will be denoted by $A$. Obviously $A$ and $K$ are disjoint, and so $A \cup K$ is a 2-component link in $S^{3}$. The ordered link $A \cup K$ will be called the factor link of $\mathcal{K}$, and the knot $\mathcal{K}$ will be called the covering knot of $A \cup K$.

A 2-component link whose first component is $A$ is a factor link of a $p$-periodic knot if and only if its linking number is relatively prime to $p$. Indeed, the preimage of the second component (call it $K$ ) is a circle in the the $p$-fold cyclic cover of $S^{3}$ 
branched along $A$ if and only if $\operatorname{lk}(A, K)$ and $p$ are coprime. Thus we can consider a link of the form $A \cup K$ as a factor $\operatorname{link}$ if $\operatorname{lk}(A, K)$ is relatively prime to $p$. Moreover, it is easy to see that $\operatorname{lk}(A, K)=\operatorname{lk}(\mathcal{A}, \mathcal{K})$ and so the linking number of a $p$-periodic knot $\mathcal{K}$ and its axis $\mathcal{A}$ is relatively prime to $p$.

We will investigate properties of factor links in order to study periodic knots.

Modification of factor knots by surgery. Let $A \cup K$ be a factor link, and consider the $(1 / n)$-surgery along $A$. The result of the surgery is again $S^{3}$, and the image $K^{\prime}$ of $K$ in the result of surgery is again a knot, but $K^{\prime}$ is not equivalent to $K$ in general. We use a sign convention so that the $(1 / n)$-surgery is described as follows. Identify the exterior of $A$ with an oriented solid torus, and let $r$ be an autohomeomorphism of the exterior which is the $(-1)$-twisting autohomeomorphism of the solid torus. Then $K^{\prime}=r^{n}(K)$, where $r^{n}$ is the composition of $n r^{\prime}$ s.

We say that two links $L_{0}, L_{1}$ are homology concordant if there is a 4-manifold $W$ with two boundary components $\Sigma_{0}, \Sigma_{1}$ which are diffeomorphic to $S^{3}$ and a proper submanifold $C$ which is diffeomorphic to $L_{0} \times[0,1]$ in $W$ such that $H_{*}\left(W, \Sigma_{0}\right)=$ $H_{*}\left(W, \Sigma_{1}\right)=0$ and $\Sigma_{0} \cap C=-L_{0}, \Sigma_{1} \cap C=L_{1}$. In other words, $L_{0}$ and $L_{1}$ are homology concordant if there is a concordance in a homology $S^{3} \times[0,1]$.

The following result will be used to detect that two periodic knots are not equivariantly concordant.

Theorem 2. If $A \cup K_{0}$ and $A \cup K_{1}$ are factor links of two equivariantly concordant periodic knots $\mathcal{K}_{0}$ and $\mathcal{K}_{1}$, then for all n, $A \cup r^{n}\left(K_{0}\right)$ and $A \cup r^{n}\left(K_{1}\right)$ are homology concordant.

Proof. Let $\mathcal{C}$ be an equivariant concordance between $\mathcal{K}_{0}$ and $\mathcal{K}_{1}$. Then there is a $p$-periodic action $f$ on $S^{3} \times[0,1]$ whose restrictions on $S^{3} \times t$ is the $\mathbf{Z}_{p}$-action of $\mathcal{K}_{t}$, for $t=0,1$, and $f(\mathcal{C})=\mathcal{C}$. The fixed point set $\operatorname{Fix}(f)$ of $f$ is diffeomorphic to $S^{1} \times[0,1]$, and is disjoint to $\mathcal{C}$.

Let $\pi: S^{3} \times[0,1] \rightarrow\left(S^{3} \times[0,1]\right) / f$ be the quotient map. Let $U$ be a tubular neighborhood of $\pi(\operatorname{Fix}(f))$, and identify $U$ with $A \times D^{2} \times[0,1]$ so that it is an extension of a null-framing along $A \times 0$ in $\left(S^{3} \times 0\right) / f \cong S^{3}$. Let

$$
\begin{aligned}
& X=\left(\left(S^{3} \times[0,1]\right) / f\right)-\operatorname{int} U, \\
& Y=X \cap\left(\left(S^{3} \times 0\right) / f\right), \\
& \tilde{X}=\pi^{-1}(X), \quad \tilde{Y}=\pi^{-1}(Y) .
\end{aligned}
$$

We need the fact that $H_{*}(\tilde{X}, \tilde{Y})=0$ implies $H_{*}(X, Y)=0$. Since we did not find this relative version in the literature, we give a proof using spectral sequences as follows. The second terms of the spectral sequence $\left\{E_{i, j}^{r}\right\}$ for the covering projection $\tilde{X} \rightarrow X$ is given by

$$
E_{i, j}^{2} \cong H_{i+j}\left(\mathbf{Z}_{p} ; H_{j}(\tilde{X})\right) \Rightarrow H_{i+j}(X) .
$$

The spectral sequence for $\tilde{Y} \rightarrow Y$ is isomorphic to $\left\{E_{i, j}^{r}\right\}$, since $H_{*}(\tilde{Y}) \cong H_{*}(\tilde{X})$. Therefore $H_{*}(X) \cong H_{*}(Y)$.

Let $h_{n}$ be an attaching homeomorphism on $A \times S^{1}$ such that $Y \cup_{h_{n}} A \times D^{2}$ is the $(1 / n)$-surgery along $A$. Let $W$ be the space $X \cup_{h_{n} \times \text { id }} A \times D^{2} \times[0,1]$. Then $\partial W$ has two components $\Sigma_{0}$ and $\Sigma_{1}$, which are the result of the $(1 / n)$-surgery along $A$. By Mayer-Vietoris arguments for $W=X \cup\left(A \times D^{2} \times[0,1]\right)$ and $\Sigma_{0}=Y \cup\left(A \times D^{2} \times 0\right)$, $H_{*}(W) \cong H_{*}\left(\Sigma_{0}\right)$, and similarly $H_{*}(W) \cong H_{*}\left(\Sigma_{1}\right)$. This proves that the union of 
$\pi(\mathcal{C})$ and $A \times 0 \times[0,1]$ in $W$ is a homology concordance between $A \cup r^{n}\left(K_{0}\right)$ and $A \cup r^{n}\left(K_{1}\right)$

Corollary 1. If $K$ is the factor knot of an equivariant slice knot, then for all $n$, $r^{n}(K)$ bounds a properly embedded 2-disk in a homology 4-ball.

In the proof of Theorem 2, we have shown that the manifold $W$ obtained by performing $(1 / n)$-surgery on $\left(S^{3} \times[0,1]\right) / f$ along $\pi(\operatorname{Fix}(f))$ is a homology $S^{3} \times[0,1]$. When $f$ is the product of the standard action on $S^{3}$ and $\operatorname{id}_{[0,1]}, W$ is diffeomorphic to $S^{3} \times[0,1]$. Futhermore, if $\left(S^{3} \times[0,1]\right) / f$ is again $S^{3} \times[0,1]$, then $\pi_{1}(W) \cong$ $\pi_{1}\left(\Sigma_{0}\right) \cong 0$ and therefore $W$ is homeomorphic to $S^{3} \times[0,1]$ by Freedman's results. The examples of nonstandard actions in [6] are of this kind.

However, the authors do not know whether there is a nonstandard action $f$ such that $W$ is not homotopic to $S^{3} \times[0,1]$, and this is why the notion of homology concordance is introduced. Fortunately the Casson-Gordon invariant vanishes for knots which bound a 2-disk in a homology 4-ball, and we will detect nonsliceness using this fact.

\section{EXAMPLES AND COMPUTATIONS}

Let $A \cup K$ be the factor link in Figure 1. We will show that for some $K_{1}$, the 2 -periodic knot $\mathcal{K}$ obtained by taking a double cover branched along $A$ satisfies all the known necessary conditions of $[14,4]$ for a periodic knot to be equivariantly slice, but is not equivariantly slice. First of all, note that $K$ is unknotted and the linking number of $A$ and $K$ is +1 .

The covering 2-periodic knot $\mathcal{K}$ of $A \cup K$ is illustrated in Figure 2. $\mathcal{K}$ bounds an equivariant Seifert surface of genus 1 , and the elements $[x],[y]$ represented by the curves $x, y$ in Figure 2 generate the first homology of the Seifert surface. The $K_{1}$ parts in Figures 1 and 2 introduce no additional twistings on the obvious bands so

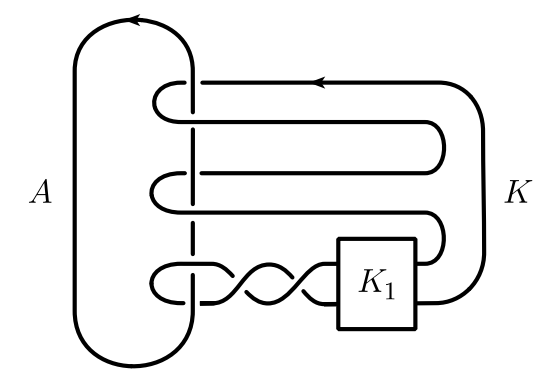

FIGURE 1

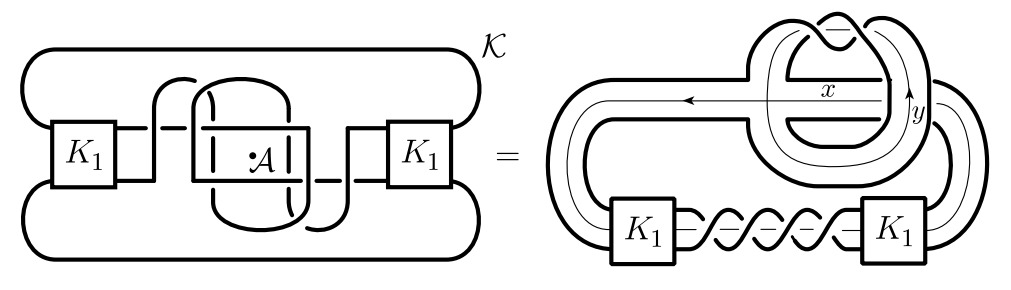

FiguRE 2 
that the linking number of $x$ in Figure 2 and a slight push-off of $x$ along the normal direction of the Seifert surface is -2 .

One can easily verify that $[x]+[y]$ generates a metabolizer $H$. Since $x$ and $y$ are left invariant under the $\mathbf{Z}_{2}$-action, $H$ is an equivariant metabolizer in the sense of [14] and [4], where $x, y$ are the curves illustrated in Figure 2.

Now we will consider the Casson-Gordon invariant of $\mathcal{K}$. In $[8,9,10]$, the values of the Casson-Gordon invariant of a knot of genus one for preferred characters are explicitly computed; the values are completely determined from the Seifert form of a generator of a metabolizer, viewing it as a knot. In the case of our example, the generator $[x]+[y]$ is represented by $K_{1} \# K_{1}$ (\# denotes the connected sum operator). Therefore, if $K_{1}$ is algebraically 2-torsion, then the Casson-Gordon invariants vanish. We will express this argument more explicitly as follows.

We make use of the following notations of $[8,9,10]$. Let $J$ be a knot and $A$ be a Seifert matrix of $J$. Let $B_{d}(J)$ be the $d$-fold cyclic cover of $S^{3}$ branched along $J$. Let $m(J)=\left(\sqrt{\left|\operatorname{det}\left(A+A^{T}\right)\right|}-1\right) / 2$. If $J$ is algebraically slice, $m(J)$ is an integer. For a complex number $z$, let $A_{z}=(1-z) A+(1-\bar{z}) A^{T}$. Define $w_{k / d}(J)$ to be the element of the Witt group $W\left(\mathbf{Q}\left(\zeta_{d}\right)\right)$ represented by $A_{\zeta_{d}^{k}}$ where $\zeta_{d}=e^{2 \pi i / d}$.

Theorem 3 (Gilmer [8, 9]). Suppose that $J$ is a knot with a Seifert surface $F$ of genus one, and $J_{x}$ is a simple closed curve on $F$ representing an element $x$ of $H_{1}(F)$ which generates a metabolizer of the Seifert pairing of J. Let $d$ be a prime power divisor of $(m(J)+1)^{q}-m(J)^{q}$ for some $q$, and $c$ be an element of $\mathbf{Z}_{d}^{*}$, the multiplicative group of units of $\mathbf{Z}_{d}$. Let $T$ be the subgroup of $\mathbf{Z}_{d}^{*}$ generated by $m(K)^{*}(m(K)+1)$, where $m(K)^{*}$ is the arithmetic inverse of $m(K)$ modulo $d$. Then, $x \otimes(c / d) \in H_{1}(F) \otimes \mathbf{Q} / \mathbf{Z}$ represents a character on $H_{1}\left(B_{d}(J)\right)$ of order $d$ in the sense of [8], and the Casson-Gordon invariant for the character is given by

$$
\tau\left(J, x \otimes \frac{c}{d}\right)=\rho\left(\sum_{k \in c T} w_{k / d}\left(J_{x}\right)\right)
$$

where $\rho$ is the map on Witt groups induced by the inclusion $\mathbf{Q}\left(\zeta_{d}\right) \rightarrow \mathbf{Q}\left(\zeta_{d}, t\right)$.

We note that $\rho$ is injective [9].

Returning to our example, suppose that $K_{1}$ is an algebraically 2-torsion knot. That is, the block sum of two copies of a Seifert matrix of $K_{1}$ admits a metabolizer. Recall that an equivariant metabolizer $H$ of the Seifert form of $\mathcal{K}$ is generated by $[x]+[y]$. The homology class is represented by a knot $J^{\prime}$, which is isotopic to $K_{1} \# K_{1}$. Since $K_{1}$ is algebraically 2 -torsion, $J^{\prime}$ is algebraically slice, and $w_{k / d}\left(J^{\prime}\right)=$ 0 for all $d$ and $k$. Applying Theorem 3 with $J_{x}=J^{\prime}$, it is proved that the CassonGordon invariants for the characters corresponding to the metabolizer $H$ always vanish. Thus, the method in [14] that uses the Casson-Gordon invariants fails to detect that $\mathcal{K}$ is not equivariantly slice.

In order to show that $\mathcal{K}$ is not equivariantly slice, we consider the result of $(-1)$-surgery as discused earlier. The knot $r^{-1}(K)$ is shown in Figure 3.

We will use the Casson-Gordon invariant to show that $r^{-1}(K)$ is not a slice knot. Note that $r^{-1}(K)$ is also obtained by tying $K_{1}$ on a handle of a Seifert surface of the stevedore's knot. But in contrasts with the case of $\mathcal{K}$, only one $K_{1}$ is tied, and the Casson-Gordon invariant of $r^{-1}(K)$ is determined by the Seifert pairing of $K_{1}$, not of $K_{1} \# K_{1}$. Therefore, for some suitable $K_{1}$ 's which are algebraically 2 -torsion but not algebraically slice, the Casson-Gordon invariant of $\mathcal{K}$ vanishes but that of $r^{-1}(K)$ does not vanish. 


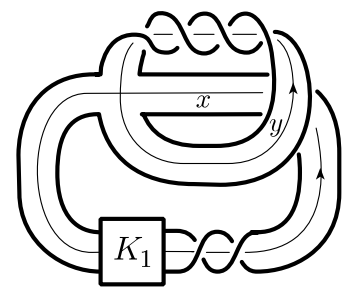

Figure 3

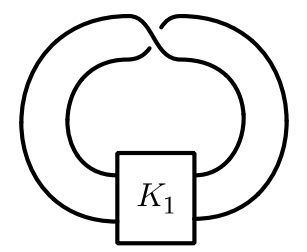

FiguRE 4

We will use Theorem 3 to compute the Casson-Gordon invariant of $r^{-1}(K)$ and will consider its discriminant as in [10]. Let $N=\left\{z \bar{z} \mid 0 \neq z \in \mathbf{Q}\left(\zeta_{d}\right)\right\} \subset \mathbf{Q}\left(\zeta_{d}\right)^{*}$. The map sending an $a \times a$ matrix $A$ to $(-1)^{a(a+1) / 2} \operatorname{det} A$ induces a map

$$
\text { dis: } W\left(\mathbf{Q}\left(\zeta_{d}\right)\right) \rightarrow \mathbf{Q}\left(\zeta_{d}\right)^{*} / N \text {. }
$$

The following is an immediate corollary of Theorem 3 .

Corollary 2. Let $J, c, d, T, x, J_{x}$ be as in Theorem 3, and let $A$ be a Seifert matrix of $J_{x}$. Then the discriminant is given by

$$
\operatorname{dis}\left(\rho^{-1} \tau\left(J, x \otimes \frac{c}{d}\right)\right)= \pm\left(\prod_{k \in c T} \operatorname{det} A_{\zeta_{d}^{k}}\right) N .
$$

The following lemma offers a practical method to detect elements of $N$. Let $d$ be a positive integer. An integer $k$ is called a $d$-norm if for every prime factor $p$ of $k$ which is coprime to $d$ and has odd exponents in $k, p$ has odd order in $\mathbf{Z}_{d}^{*}$.

Lemma 1 (Gilmer-Livingston [10]). For odd $d, k$ is a d-norm if and only if $k \in N$.

For $r^{-1}(K)$, there are two metabolizers which are generated by the homology classes $u_{1}=[x]-[y]$ and $u_{2}=2[x]+[y]$, respectively. The former is represented by a curve which is isotopic to $K_{1}$ as a knot in $S^{3}$. The latter is represented by a curve isotopic to a knot shown in Figure 4. Denote it by $K_{2}$.

\section{Lemma 2.}

$$
\operatorname{dis}\left(\rho^{-1} \tau\left(r^{-1}(K), u_{1} \otimes \frac{c}{d}\right)\right)= \pm \operatorname{dis}\left(\rho^{-1} \tau\left(r^{-1}(K), u_{2} \otimes \frac{c}{d}\right)\right) .
$$

Proof. Define the Alexander polynomial of a $2 a \times 2 a$ matrix $A$ to be

$$
\Delta_{A}(t)=\left(1 / t^{a}\right) \operatorname{det}\left(t A-A^{T}\right) .
$$

If $|w|=1$,

$$
\begin{aligned}
\operatorname{det} A_{w}=\operatorname{det}((\bar{w}-1)(w A & \left.\left.-A^{T}\right)\right)=\left(w(\bar{w}-1)^{2}\right)^{a} \Delta_{A}(w) \\
& =(-(1-w)(1-\bar{w}))^{a} \Delta_{A}(w) \equiv \pm \Delta_{A}(w) \bmod N .
\end{aligned}
$$


Let $A$ be a Seifert matrix of $K_{1}$. Then the matrix

$$
\left[\begin{array}{cc}
A & A \\
A^{T} & A
\end{array}\right]
$$

is a Seifert matrix of $K_{2}$ (e.g. see [3]). Denote it by $i_{2}(A)$ as in [3]. By a theorem of [3], the Alexander polynomial $\Delta_{i_{2}(A)}(t)$ of the Seifert matrix $i_{2}(A)$ is equal to $\Delta_{A}\left(t^{2}\right)$. It can be also checked by a direct computation.

Since $T$ is the the multiplicative subgroup of $\mathbf{Z}_{d}^{*}$ generated by $2,2 T=T$. Hence,

$$
\begin{aligned}
\prod_{k \in c T} \operatorname{det}\left(i_{2} A\right)_{\zeta_{d}^{k}} & \equiv \pm \prod_{k \in c T} \Delta_{i_{2} A}\left(\zeta_{d}^{k}\right)= \pm \prod_{k \in c T} \Delta_{A}\left(\zeta_{d}^{2 k}\right) \\
& = \pm \prod_{k \in c \cdot 2 T} \Delta_{A}\left(\zeta_{d}^{k}\right)= \pm \prod_{k \in c T} \Delta_{A}\left(\zeta_{d}^{k}\right) \equiv \pm \prod_{k \in c T} \operatorname{det} A_{\zeta_{d}^{k}} \bmod N
\end{aligned}
$$

By Corollary 2, the lemma is proved.

Theorem 4. Let $q$ be a prime power, $d$ be a prime power divisor of $2^{q}-1$, and $T$ be the subgroup of $\mathbf{Z}_{d}^{*}$ generated by 2. Let $A$ be a Seifert matrix of $K_{1}$. If $\left|\prod_{k \in c T} \operatorname{det} A_{\zeta_{d}^{k}}\right| \notin N$ for some $c$, then $\mathcal{K}$ is not an equivariant slice knot.

Proof. Suppose that $\mathcal{K}$ is equivariantly slice. By results of $[1,9]$ and by Corollary 1 , $\tau\left(r^{-1}(K), u_{i} \otimes(c / d)\right)=0$ for some $i \in\{1,2\}$, since the Casson-Gordon invariant of a knot in $S^{3}$ vanishes if the knot bounds a properly embedded 2-disk in a homology 4-ball (see a remark in [7]). By Lemma 2,

$$
\operatorname{dis} \rho^{-1} \tau\left(r^{-1}(K), u_{1} \otimes(c / d)\right)= \pm\left(\prod_{k \in c T} \operatorname{det} A_{\zeta_{d}^{k}}\right) N=N .
$$

As an example, let $K_{1}$ be the figure-eight knot $4_{1}$. Let $\mathcal{K}$ be the 2-periodic knot as in Figure 2. Let $q=5, d=2^{q}-1=31, c=5 . A=\left[\begin{array}{cc}-1 & 1 \\ 0 & 1\end{array}\right]$ is a Seifert matrix of $K_{1}$, and a straightforward computation aided by a computer program shows that

$$
\left|\prod_{k \in c T} A_{\zeta_{d}^{k}}\right|=43431
$$

Since $3 \cdot 31 \cdot 467$ is the prime factorization of 43431 and 3 has order 30 in $\mathbf{Z}_{d}^{*}, 43431$ is not a $d$-norm and so 43431 is not in $N$. By Theorem $4, \mathcal{K}$ is not equivariantly slice.

Since $4_{1}$ is algebraically 2 -torsion, the periodic knot $\mathcal{K}$ satisfies all necessary conditions of Naik [14] and Choi-Ko-Song [4] as explained before. This proves Theorem 1.

In addition, note that $4_{1}$ is amphicheiral. The knot $\mathcal{K}$ can be considered as a satellite of $4_{1} \# 4_{1}$. Indeed $\mathcal{K}$ is obtained by wrapping the stevedore's knot along $4_{1} \# 4_{1}$. Since both $4_{1} \# 4_{1}$ and the stevedore's knot are slice knots, $\mathcal{K}$ is also a slice knot (without periodicity).

We remark that the above discriminant calculation shows that the figure-eight knot $4_{1}$ can be used to construct links which are not concordant to boundary links and non-slice links as in [10]. The 3 full-twisted double $T_{3}$ of the unknot was used to construct such examples in [10], but simpler examples are obtained using $4_{1}$. Of 
course, the knot $T_{3}$ can also be used as $K_{1}$ in our construction. $T_{3}$ is algebraically 2-torsion. Indeed,

$$
\left[\begin{array}{cccc}
-1 & 1 & 0 & 0 \\
0 & 3 & 0 & 0 \\
0 & 0 & -1 & 1 \\
0 & 0 & 0 & 3
\end{array}\right]
$$

is the a Seifert matrix of $T_{3} \# T_{3}$, and the vectors $\left[\begin{array}{llll}-1 & 1 & 1 & 0\end{array}\right]$ and $\left[\begin{array}{llll}1 & 0 & 2 & 1\end{array}\right]$ generate a metabolizer. Therefore all necessary conditions in $[14,4]$ are satisfied as before. However, in [10], it was shown that some discriminants of $T_{3}$ are nontrivial and so the 2-periodic knot $\mathcal{K}$ corresponding to $T_{3}$ is not equivariantly slice. The authors do not know whether $\mathcal{K}$ is slice (without periodicity) or not, in this case.

\section{REFERENCES}

[1] A. Casson and C. Gordon, Cobordism of classical knots, "A la recherche de la Topologie perdue", ed. by Guillou and Marin, Progress in Mathematics, Volume 62, 1986. CMP 19:16

[2] A. Casson and C. Gordon, On slice knots in dimension three, Proc. Symp. in Pure Math. XXX (1978), part two, 39-53. MR 81g:57003

[3] J. Cha. Signatures of links in rational homology spheres, preprint.

[4] D. Choi, K. Ko and W. Song, Concordance of periodic knots, preprint.

[5] D. Cooper, The universal abelian cover of a link, in Low-Dimensional Topology (Bangor, 1979), London Math. Soc. Lecture Note Ser. 48 (1982), 51-66. MR 83g:57002

[6] C. Giffen, The generalized smith conjecture, Amer. J. Math. 88 (1966), 187-198. MR 33:6620

[7] P. Gilmer, Slice knots in $S^{3}$, Quart. J. Math. Oxford 34 (1983), 305-322. MR 85d:57004

[8] P. Gilmer, Classical knot and link concordance, Comment. Math. Helv. 68 (1993), 1-19. MR 94c:57007

[9] P. Gilmer, C. Livingston, The Casson-Gordon invariant and link concordance, Topology 31 (1992), 475-492. MR 93h:57037

[10] P. Gilmer, C. Livingston, Discriminants of Casson-Gordon invariants, Math. Proc. Camb. Phil. Soc. 112 (1992), 127-139. MR 94e:57007

[11] J. Levine, Knot cobordism group in codimension two, Comm. Math. Helv. 44 (1969), 229244. MR 39:7618

[12] J. Milnor, Infinite cyclic coverings, Conference on the Topology of Manifolds, Prindle, Weber \& Schmidt, Boston, Mass., 1968, pp. 115-133. MR 39:3497

[13] K. Murasugi, On periodic knots, Comment. Math. Helv. 46 (1971), 162-174. MR 45:1148

[14] S. Naik, Equivariant concordance of knots in $S^{3}$, Proceedings of Knots 96 (Edited by Shin'ichi Suzuki), World Scientific Publishing Co., 1997, pp 81-89.

Department of Mathematics, Korea Advanced Institute of Science and Technology, TAEJon, 305-701, Korea

E-mail address: jccha@knot.kaist.ac.kr

E-mail address: knot@knot.kaist.ac.kr 